\title{
An Improved Otsu Method using the Weighted Object Variance for Defect Detection
}

\author{
Xiao-cui Yuan ${ }^{\mathrm{a}}$, Lu-shen $\mathrm{Wu}^{\mathrm{a}} *$ and Qingjin Peng ${ }^{\mathrm{b}}$ \\ ${ }^{a}$ School of Mechanical and Electrical Engineering, Nanchang University, Nanchang, 330031, PR China \\ ${ }^{b}$ Department of Mechanical Engineering, University of Manitoba, Winnipeg, R3T 5V6, Canada
}

\section{ABSTRACT}

Defects on product surfaces affect quality of the product. Machine vision provides an efficient tool for the surface defect detection. Threshold is commonly used to separate objects from the image background in the vision-based inspection method. The Otsu method is one of the most used approaches to decide the threshold for a satisfied result when the image histogram is bimodal, but it fails when the histogram of an image is unimodal or close to unimodal. Defects in product surfaces can range from small to large sizes, which results in distributions of the image histogram change from unimodal to bimodal. An improved Otsu method, named the weighted object variance (WOV), is proposed in this research to detect defects on product surfaces. A parameter that equals the cumulative probability of defects occurrence is weighted on the object variance of between-class variance. The weight ensures that the threshold always be a value that locates at the valley of two peaks or at the left bottom rim of a single peak histogram. It is essential to have a high detection rate and low false alarm rate for the defect detection. Experimental results demonstrate the effectiveness of the improved Otsu method in the defect detection of various surfaces. Compared to other thresholding methods such as maximum entropy, Otsu, valley-emphasis, and modified valley-emphasis methods, the WOV method provides better segmentation results.

Key words: Image segmentation; Otsu thresholding; Defects detection; Machine vision;

2015 Elsevier Ltd. All rights reserved. 


\section{Introduction}

Defects on product surfaces reduce the product quality and the commercial value. A lot of efforts have been made to inspect these defects. The manual inspection based on human vision is time-consuming. Non-destructive testing methods provide efficient ways for the defect detection, such as methods of penetrant testing (PT), magnetic particle (MP)[1], eddy current (EC)[2] and machine vision inspection[3]. PT can find extremely fine flaws on product surfaces, but results are poor for the rough or dirty surfaces. MP can accurately find surface cracks, but it can only be used for ferromagnetic parts. EC can fast detect defects of conducting materials. However, its detection result is very sensitive to the liftoff of probes. Inspection of surface defects using machine vision has a relatively high speed and accuracy, it has been widely applied by manufacturing enterprises[4, 5]. Three processes are required in a vision-based inspection system including image acquisition, image processing, and defect conclusion.

Image processing analyzes the characteristic of images to find defects, which includes steps of image segmentation, feature extraction, and defects classification. Image segmentation is one of the most important and challenging procedures in the defect detection. Thresholding is a commonly used tool in the image segmentation for extracting defects from the image background[6]. Thresholding techniques can be categorized into global thresholding and local thresholding[7]. Local thresholding uses the localized gray-level information to choose multiple threshold values, it can deal with the non-uniform illumination but the process is complicated and slow. Global thresholding, using a single threshold for the entire image, is easy to implement and widely used in automatic visual inspection applications to achieve the effective and accurate detection [8]. This paper mainly discusses the global threshold for defect detection.

In the past decades, a number of thresholding methods have been proposed to detect surface defects. For example, Li et al.[9] proposed a thresholding method based on the maximum weighted object correlation for the rail defect detection. This thresholding method is an improved maximum entropy method. Funck et al.[10] used clustering and region-growing techniques to detect defects on wood images. Various defect detection applications of thresholding technologies are discussed in literatures [11-13]. Most of thresholding methods provide expected results for the particular application, but there is not a general method available for defect detection.

Sezgin [14] did a thorough survey in a variety of global thresholding techniques, and suggested that Otsu [15] thresholding is one of the most commonly used methods to decide a threshold for general images. By selecting threshold values of the maximum between-class variance or the minimum within-class variance from the image histogram, the Otsu method can obtain a satisfied segmentation when the object and image background have the similar variance, however, the method fails if sizes of the object and image background are great difference. In other words, the Otsu method provides a satisfactory result for thresholding an image with a histogram of the bimodal distribution, but it fails for the image histogram with the unimodal or close to the unimodal distribution[16]. For accurately segmenting images, some improved Otsu methods have been proposed. For example, Hu et al.[17] used the range of image background proportions to the region of interests to confine the range of the threshold selection and achieved reliable results in segmenting magnetic resonance and computed tomography images of the human brain. Qiao et al.[18] suggested a criterion using the weighted sum of the within-class variance and the intensity contrast for segmenting small objects by exploring the knowledge of the intensity contrast. For effectively detecting defects, $\mathrm{Ng}$ [19] proposed a revised Otsu method known as the valley-emphasis (VE) method using the valley point information in the objective function. The VE method can successfully detect defects in some images, but it fails for images where defects are very small. For this problem, Fan and $\mathrm{Bo}[20]$ proposed a modified VE method, named the neighborhood valley-emphasis (NVE), using the neighborhood information at the valley point to improve the segmentation quality. Although Qiao, $\mathrm{Hu}, \mathrm{Ng}$, et al. argued that the Otsu method is inappropriate in segmenting small objects from the image background as the threshold biases to the larger variance, while their results were all based on the experiment without theoretical supports. $\mathrm{Xu}$ et al.[21] discovered an important property of the Otsu threshold which is equal to the average of the mean levels of two classes partitioned by this threshold. Their discovery reasonably explains the experimental results of Qiao, $\mathrm{Hu}$ and $\mathrm{Ng}$.

The thresholding methods discussed above are onedimensional Otsu methods that only consider the gray level information without considering the spatial neighborhood information, it is difficult to obtain satisfactory segmentation results for the noise or illumination inequality image[22]. The two-dimensional (2D) Otsu method is proposed to get better segmentation results by utilizing both the gray level information of each pixel and its spatial correlation information within the neighborhood. However, it increases the computational complexity and is difficulty to use in real time vision inspection systems [23]. The improved 2D Otsu methods can be found in literatures [24, 25].

By analyzing problems of the Otsu and improved Otsu methods for defect detection applications, this paper develops an effective thresholding method, named the weighted object variance (WOV), for defect detection in vision inspection systems. The contributions of this research are summarized as follows:

(1) An improved Otsu method is proposed to weight a parameter on the object variance of between-class variance. The weight is equal to a cumulative probability of the defects occurrence to ensure that the threshold will always be a value that resides at the valley of two peaks or at the left bottom rim of a single peak histogram.

(2) The effectiveness and performance of the proposed method are validated with the misclassification error for various kinds of images and detection rate (DR), false alarm rate (FAR) of defects for thousands of rail surface images.

The remaining parts of the paper are organized as follows: In Section 2, the problems of Otsu and improved Otsu methods for defect detection are analyzed. Our method is proposed in Section 3. In Section 4, experimental results and analysis are discussed. Conclusions and future research are described in Section 5 .

\section{Problems of the existing Otsu methods for defect detection}

The Otsu method for selecting an image threshold is briefly introduced firstly in this section. The problems of Otsu and widely used improved Otsu methods, such as VE and NVE methods, in thresholding objects of images are then analyzed to propose a revised Otsu method for the defect detection.

\subsection{The Otsu method}


An image can be described as $I(x, y)$ with a gray level range from 0 to $L-1$, where $L$ is the number of distinct gray levels. Let the number of pixels with the gray-level $i$ be $n_{i}$ and $n$ be the total number of pixels in a given image with the size of $M \times N$. The probability of occurrence of the gray level $i$ is defined as follows.

$p_{i}=\frac{n_{i}}{n}$

If an image is divided into two classes, $D_{0}$ and $D_{1}$, by a threshold $t, D_{0}$ consists of pixels with levels $[0, t]$, and $D_{1}$ consists of pixels with levels $[t+1, L-1]$. Let $P_{0}(t)$ and $P_{1}(t)$ denote the cumulative probabilities, $u_{0}(t)$ and $u_{1}(t)$ denote the mean levels of $D_{0}$ and $D_{1}$ classes, respectively.

$$
\begin{aligned}
& P_{0}(t)=\sum_{i=0}^{t} p_{i} \\
& P_{1}(t)=\sum_{i=t+1}^{L-1} p_{i}=1-P_{0}(t)
\end{aligned}
$$

The mean levels of the two classes can be computed as:

$$
\begin{aligned}
& u_{0}(t)=\sum_{i=0}^{t} i * \frac{p_{i}}{P_{0}(t)} \\
& u_{1}(t)=\sum_{i=t+1}^{L-1} i * \frac{p_{i}}{P_{1}(t)}
\end{aligned}
$$

For a threshold $t$, Otsu shows that the between-class variance $\delta_{b}(t)$ of $D_{0}$ and $D_{1}$ is as follows.

$$
\delta_{b}(t)=P_{0}(t)\left(u_{0}(t)\right)^{2}+P_{1}(t)\left(u_{1}(t)\right)^{2}
$$

The optimal threshold $T H$ of Otsu method can be determined as:

$$
\begin{aligned}
T H & =\arg \max _{1<t<L} \delta_{b}(t) \\
& =\arg \max _{1<t<L}\left(P_{0}(t)\left(u_{0}(t)\right)^{2}+P_{1}(t)\left(u_{1}(t)\right)^{2}\right)
\end{aligned}
$$

In the case of a single threshold, the Otsu method works well for images with the histograms bimodal distribution. However, the threshold value will be incorrect when the histogram is the unimodal or close to the unimodal distribution. In vision inspection applications, most products are defect-free, or some products contain defects, but the defects are much small comparing with the defect-free areas. Thus, the histograms of images are unimodal or close to unimodal distributed. The reason for the Otsu method failing to segment images with the histogram unimodal distribution is that the Otsu threshold is equal to the average of the mean levels of two classes partitioned by this threshold[21].

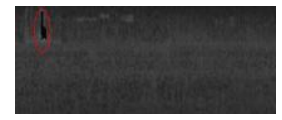

(a)

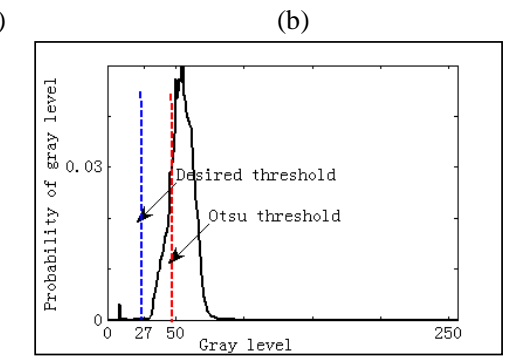

(d)

Fig.1 Problem of the Otsu method in thresholding small defects: (a) Original image with a small defect; (b) Otsu thresholding result; (c) Desired threshold result; (d) Histogram and threshold values.
Fig. 1 (a) shows a rail image with one defect (dark area in the red circle). The defect is very small compared to the size of the image background. The histogram of the image is close to the unimodal distribution as shown in Fig. 1 (d). An incorrect threshold is generated using the Otsu method, the segmentation result of Fig. 1 (a) is shown in Fig. 1 (b), while the desired result is shown in Fig. 1(c). A comparison of the desired threshold and the Otsu threshold is shown in Fig. 1 (d). In this paper, the area with the gray level lower than the threshold is defined as objects or defects (black pixels), gray levels of pixels that are higher than the threshold is defined as the background (white pixels).

\subsection{The improved Otsu methods}

The Otsu method can obtain a satisfied segmentation only when the object and image background have the similar variance. In order to automatically and correctly detect defects using the Otsu method, many improved Otsu methods were proposed. Such as $\mathrm{Ng}$ [19] and Fan [20] introduced valley-emphasis methods (VE and NVE) which keep threshold values locating at the valley of histogram. $\mathrm{Ng}$ [19] and Fan [20] mainly changed Eq. (6) into Eq. (8) as follows:

$$
\beta_{b}(t)=(1-p(t))\left(P_{0}(t)\left(u_{0}(t)\right)^{2}+P_{1}(t)\left(u_{1}(t)\right)^{2}\right)
$$

where $1-p(t)$ is the weight of between-class $\delta_{b}(t)$ in Eq. (6). $p(t)$ is the sum of the neighborhood gray probability in interval $n=2 m+1$ for the gray level $t$, where $n$ is the neighborhood length. Fan [20] suggested that $n=11$ is a better selection than other values. When $n=1$, the NVE method is equal to the VE method. The VE method selects a threshold value closing to the valley of histogram, and the segmentation result is better than Otsu's result. The VE method works well for some defective images with large defects. However, it incorrectly detected some defect-free images to defective images. The NVE method improved the VE method using the neighborhood information at the valley point to improve the segmentation quality. In our experiment, it is found that only if the histogram of an image is bimodal or close to bimodal, the NVE method can provide a satisfied result. If the histogram of an image is unimodal, the NVE method fails as its threshold value locates at the bottom rim (right bottom rim or left bottom rim) of the histogram. In other words, the NVE method can provide satisfied results for most defective images, but fails to segment some defect-free images.

Fig. 2 shows segmentation results of the VE and NVE methods for three images that contain two defect-free images (images I and II) and one defective image (image III). Columns (a) to (c) are original images, segmentation results of the VE and NVE methods, respectively. Fig .3 is the image histogram corresponding to Fig. 2 (a). The NVE method provides completely wrong thresholds for both images I and II with threshold $T H=147$ and $T H=213$ respectively, which segments almost all background as the objects. The NVE threshold value locates at the right rim of the histogram, but the desired threshold should be in the left rim as shown in Figs. 3 (a) and 3 (b). The VE threshold value locates at the local valley of the histogram, and segments parts of the background as objects.

Research on automatic thresholding for the defect detection, such as the VE and NVE methods, mainly focuses on the threshold selection for defective images. The defect-free image segmentation is neglected, which results in that the most thresholding methods can correctly separate defects from their background, but wrongly conclude a defect-free image into the detective image. If a defect-free image is detected as the defective image, it will lead to a false defect in the detection. An 
ideal vision inspection system should have a high DR and low FAR of defects [9].

Generally, the gray level of defect is different from the image background or the defect-free area. For example, in Fig. 1 (a) and image III in Fig. 2 (a), defects are in the red circles and their gray levels are lower than the image background. The defect can be detected according to its gray level information. However, it is a challenge to select an optimal threshold to separate defects from the image background because of the following factors:

(1) Some product surfaces are often illumination inequality and their images are low contrast between defects and the background.

(2) Most of product surfaces have no defects or the defect is much smaller than its background area, thus, the image histogram is unimodal or close to a unimodal distribution.

(3) Some products are exposed outside environments, their defects are easily hidden in the environments. For example, in the detection of rail surface defects, the defects are often hidden in rust.

By analyzing the problems and challenges in existing thresholding methods for defect detection, this paper proposes an improved Otsu method for the defect detection aiming at obtaining a high DR and low FAR of defects in vision inspection systems.

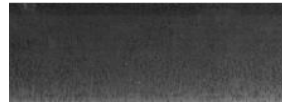

Image I

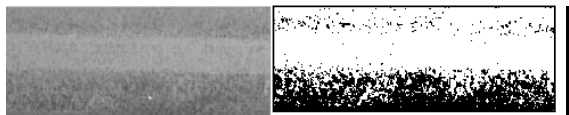

Image II

$T H=130$

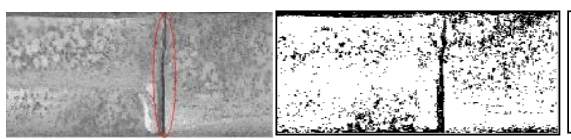

Image III

(a)

$T H=134$

(b)

$T H=69$

(c)

Fig. 2 Segmentation results of defective and defect-free images, from the left to right columns are original images, the segment results for the VE and NVE methods, respectively.

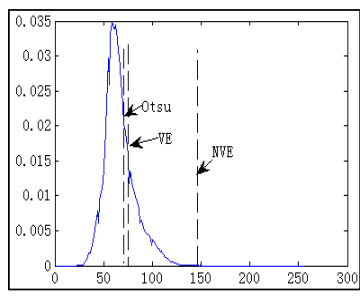

(a)

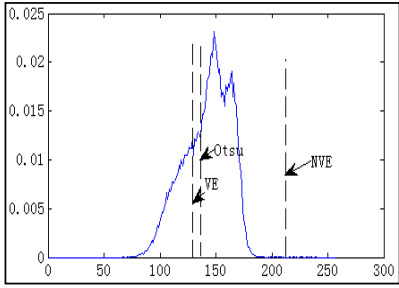

(b)

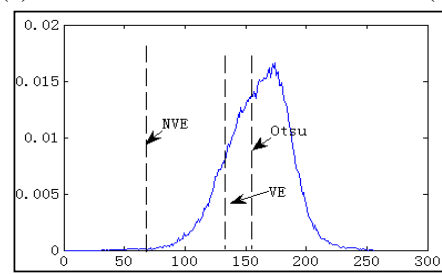

(c)

Fig.3 (a)-(c): The histogram and threshold values of image I to image III of Fig.2 (a)

\section{Proposed method}

\subsection{The proposed WOV method}

From the above discussion of the Otsu method, the first item in Eq. (6), $P_{0}(t)\left(u_{0}(t)\right)^{2}$, is defined as the object variance and the second item $P_{1}(t)\left(u_{1}(t)\right)^{2}$ is the background variance. If the threshold locates at the left bottom rim of a single peak, or at the valley of two peaks (the first peak is much smaller than the second peak), the object variance is close to 0 and the background variance is close to maximum. When increasing the gray level $t$, the object variance increases, but the background variance decreases. When the gray level $t=\left(u_{0}(t)+u_{1}(t)\right) / 2$, the between-class variance reaches maximum[21]. Fig. 4 (a) shows the gray level variance of Fig. 1 (a). When $t<30$, the object variance is close to 0 , and the background variance is close to maximum. The desired threshold of Fig. 1 (a) should be smaller than 30 , but the Otsu threshold is close to 50 .

In the case of the gray level of the defect is lower than its background, the desired threshold should be located at the left bottom rim of the histogram for a defect-free image or the valley between the small peak and large peak of the histogram for the defective image. In defect detection applications, most images are defect-free. Even products contain defects, the defects area is normally much smaller than the background area. Thus, the histograms of defective images are close to the unimodal distribution. Therefore, the desired threshold should be close to a low gray level comparing with the threshold generated by the Otsu threshold. The Otsu threshold $T H=\left(u_{0}(t)+u_{1}(t)\right) / 2$ makes the object and background have a similar size [21]. The desired threshold should keep the probability of defects in a small value or even equal to 0 . In order to get a desired threshold, the first item (the object variance) should contribute less for the between-class variance, and the threshold should be mainly decided by the second item (the background variance). A parameter $\omega$ ranging from 0 to 1 can be weighted on the first item, and the second item is kept unchanging. The weighted object variance is $\omega$ times of the original object variance. The proposed method can be written as follows.

$Y_{b}(t)=\omega P_{0}(t)\left(u_{0}(t)\right)^{2}+P_{1}(t)\left(u_{1}(t)\right)^{2}$

The optimal threshold $T H$ of the proposed method is:

$$
\begin{aligned}
T H & =\arg \max _{1<t<L} \mathrm{Y}_{b}(t) \\
& =\arg \max _{1<t<L}\left(\omega P_{0}(t)\left(u_{0}(t)\right)^{2}+P_{1}(t)\left(u_{1}(t)\right)^{2}\right)
\end{aligned}
$$

In Eq. (9), the parameter $\omega$ is a weight of the object variance, and the value of $\omega$ is ranged from 0 to 1 . As $\omega P_{0}(t)\left(u_{0}(t)\right)^{2} \leq P_{0}(t)\left(u_{0}(t)\right)^{2}$, the threshold value of the proposed method is equal to or smaller than the Otsu's threshold. As $\omega$ is ranged from 0 to 1 , we select $\omega=0.1$ randomly as an example of the weighted gray variance and use Fig. 1 (a) to illustrate the variation of the weighted gray variance. In Fig.4 (b), the weighted object variance (blue curve) of Fig.1 (a) is much smaller than the original object variance (green curve) to contribute less for the between-class variance. The curves of $\delta_{b}(t)$ and $Y_{b}(t)$ in Fig. 1 (a) are shown in Fig. 4 (c). Therefore, the resulted threshold is mainly decided by the background variance, and close to the desired threshold. In a vision inspection system, the image varies greatly from different products, and a fixed weight value cannot satisfy the application 
requirement. Therefore, an automatic weighting method is needed to select an optimal threshold for the image segmentation.

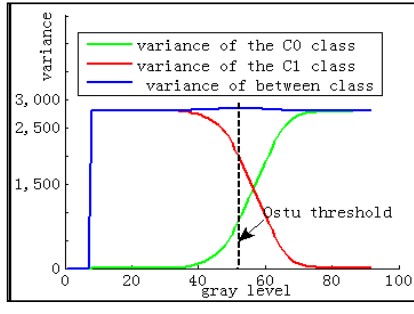

(a)

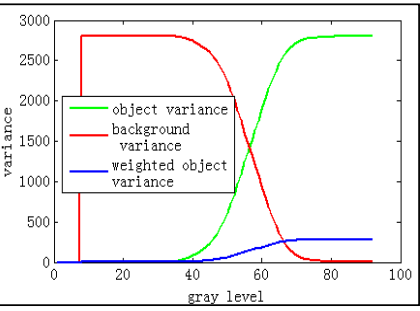

(b)

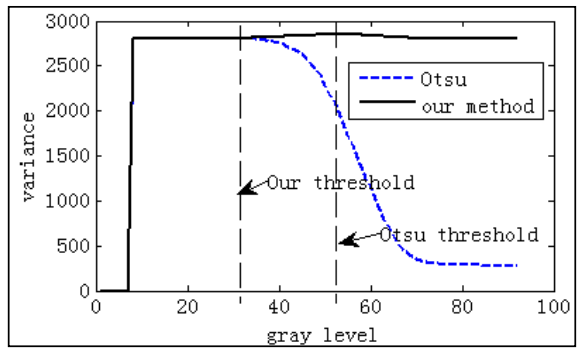

(c)

Fig.4 Gray variance of Fig.1 (a): (a) Curves of gray variance of Fig.1 (a); (b) Comparison of before and after weighted object variance with $\omega=0.1$; (c) curves of $\delta_{b}(t)$ and $Y_{b}(t)$ with $\omega=0.1$

\subsection{Adaptive weight selection for the WOV method}

The weight $\omega$ in Eq. (9) is ranged from 0 to 1 . A smaller $\omega$ will result in a smaller threshold because the threshold will be mainly decided by the background variance. A larger $\omega$ can make the threshold close to the Otsu threshold. Different images need different $\omega$ values. Therefore, an adaptive weight is required for the vision inspection. In the case of a defect-free image, the object variance should be close to 0 , and $\omega$ can be selected to close 0 , while $\omega$ should be set as a larger value for the defective image. The large defect requires the $\omega$ value to close to 1. It would be better for the weight to vary with the probability of a defects occurrence. The cumulative probability $P_{0}(t)$ in Eq. (2) with the value range from 0 to 1 is equal to the cumulative probability of the defects occurrence when defects are segmented by $t$. The value of $P_{0}(t)$ increases with the gray level $t$ in a nonlinear fashion. When the threshold locates at the left bottom rim of the single peak for a defect-free image, $P_{0}(t)$ is close to 0 , and when the threshold value resides at the valley of two peaks for a defective image, a larger defect will result in a larger value of $P_{0}(t)$. Inspired by the value of $P_{0}(t)$, we set $\omega=P_{0}(t)$. Eq. (9) can be rewritten as follows:

$Y_{b}(t)=P_{0}(t) P_{0}(t)\left(u_{0}(t)\right)^{2}+P_{1}(t)\left(u_{1}(t)\right)^{2}$

\section{Experimental results and analysis}

Experiments are conducted in a computer using Matlab R2009a, with Intel Core $2.30 \mathrm{GHz}$ CPU and 1.19GB memory. In order to validate the effectiveness of the proposed method, three measures are applied to evaluate the thresholding performance: one is misclassification error (ME) to evaluate the segmentation result for a single image. ME reflects the percentage of background pixels wrongly assigned to the foreground, and conversely, foreground pixels are wrongly assigned to the background[26]. The others are DR and FAR of the defect detection. DR and FAR are widely used as criteria in the pattern recognition[27]. They are applied to comprehensively assess the defect detection results for multiple images. The Otsu and other methods, such as the VE, NVE and maximum entropy[28], are compared to the proposed WOV method. Among these methods, the VE and NVE are improved Otsu methods, and the maximum entropy is a classic global thresholding method that is widely used to the image segmentation for defect detection applications[29].

Three different sets of images are used to test the segmentation results. One set contains several common defects in different materials of detection applications, such as wood, metal, fabric and rail images to test the ME value. Another tested data set with 1000 rail images is used for the evaluation of DR and FAR of the defect detection. The last data set is used to test the segmentation result for other applications.

\subsection{ME values in various defect detection applications.}

For a two-class segmentation problem, ME can be simply expressed as:

$\mathrm{ME}=1-\frac{\left|B_{O} \cap B_{T}\right|+\left|F_{O} \cap F_{T}\right|}{\left|B_{O}\right|+\left|F_{O}\right|}$

where $B_{O}$ and $F_{O}$ represent the background and foreground area pixels of the manually thresholded image respectively, $B_{T}$ and $F_{T}$ denote the background and foreground area pixels in the image that are thresholded using different methods. $|\bullet|$ is the cardinality of the set. The ME value varies from 0 for a perfectly classified image to 1 for a totally wrongly binarized image. Many literatures use ME values to evaluate the segmentation result of images, such as references [30-32].

In order to test the extensive use of the proposed method, ten images including metal, wood, fabric and rail surface images are provided to be segmented. ME values are employed to measure the segmentation results. Among these images, metal, wood, and fabric images are collected in indoor. Seven rail surface images are captured in different railways with different circumstances outside. More images collected outside are included in the experiment because they are more difficult to detect defects than images collected indoor since products exposed outside are easily affected by the environment. As the railway exposes outside, some rail surfaces are covered by a lot of rust where the image gray levels are usually lower than their gray levels of background and are easily detected as defects (such as images I and II in Figs. 8 and 9). Some railway surfaces are smooth and used frequently, and the center regions of these rail surfaces are often smooth to reflect more light by the specular reflection than periphery regions covered by rust. Therefore, the gray levels of the center region are higher than the periphery region (Such as images III, IV in Figs. 10 and 11). Except images with defects to be segmented, the segmentation of defect-free images is also very important in vision inspection systems. Therefore, the defect-free image segmentation is included in our experiment. Figs. 5 to 14 show segmentation results of the five methods and histograms of the images. Table 1 displays ME values of ten images using the five methods. The dark areas in red circles are defects.

Fig.5 (a) is a metal image with two small defects. The Otsu method provides a bigger threshold than others and segments a part of the background into the defect. Although the NVE method provides a ME value that is close to 0 , it wrongly segments all pixels to the background with threshold $T H=62$. The VE and maximum entropy methods obtain perfect results 
with $\mathrm{ME}$ values equaling 0 . The proposed method generates a threshold value $T H=76$, and misses one defect. Fig.6 (a) is a wood image with one defect to be detected. The Otsu and VE methods obtain the same threshold, and they improperly segment more than a half of the background to defects with ME values equaling 0.6894. Fig. 7 shows an example of the contamination detection on the fabric image. Both our and maximum entropy methods obtain an ideal threshold. The NVE method gives a wrong result that segments two small defects into the background.

Figs. 8 to 10 show the segmentation results of three defectfree rail images. Two defect-free images (images I and II in Figs. 8-9) are covered by rust, and the other one is smooth in center but dark in periphery (image III in Fig.10). The NVE method perfectly segments the rail image I, in which the threshold value locates at the left bottom rim of histogram, but provides fully an incorrect result for image II, in which the threshold value locates at the right bottom rim of histogram, and it segments all the background into the defect. The Otsu, VE and maximum entropy methods provide incorrect results for both images I and II, and the results of image I are worse than image II. The NVE and our methods obtain perfect results for image III, but other three methods wrongly segment the dark periphery into defect.

Figs. 11-14 show the segmentation results of four defective rail images. Two rail surfaces are smooth (images IV and $\mathrm{V}$ in Figs. 11-12) while the others are covered by rust (images VI and VII in Figs. 13-14). Image IV contains many slight scratches and a large defect which is indentation. For image IV, the center region is smooth but the periphery area is dark. The NVE method segments all the defects to the background as its threshold locates at the left bottom rim of histogram. The maximum entropy method detects the large defect well, but wrongly segments periphery into defects. Our method does not detect the background as defects, but wrongly segments a part of defects to background. The Otsu and VE methods obtain large thresholds that correctly segment the large defect and some scratches, however, parts of background are wrongly segmented into defects. Rail images V-VII contain cracks on rail surfaces, but the rail surface of image $\mathrm{V}$ is smooth with the uniform illumination. Images VI and VII covered by rust, image VII looks very dark. Except the Otsu and VE methods, other three methods obtain acceptable results for images V and VI. For image VII, the VE and our methods provide perfect results, but NVE and maximum entropy methods almost generate completely wrong results with ME near to 1.

It can be concluded from this group of experiments, the VE method provides a threshold value located at the local valley of histogram, thus it obtains incorrect results for most of defect-free images. The NVE method usually generates a threshold value located at the bottom rim of histogram. Therefore, it segments almost all background to defects or defects into background. Maximum entropy method gives good results for some defective images but fails to inspect defect-free images. As the weight on the object variance of between-class equals the cumulative probability of defects occurrence, the proposed WOV method always makes the probability of defects occurrence in a small value and provides a threshold located at the left bottom rim of a single peak, or at valley of two peaks, therefore, it presents nearly a perfect segmentation result for various different images in applications of defect detection. From Table 1, it can be seen that ME values generated by the WOV method are all close to 0 .

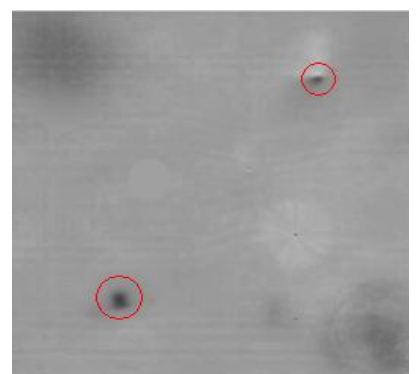

(a)

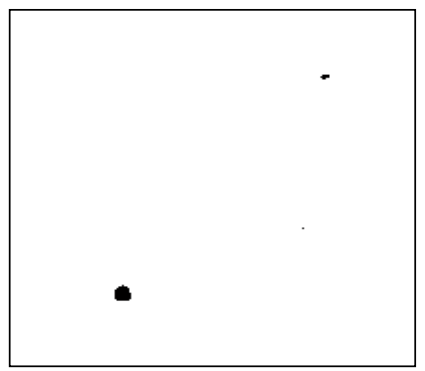

(e)

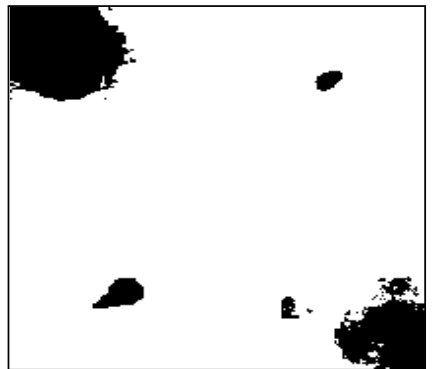

(b)

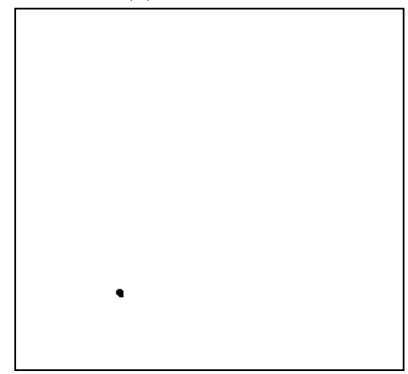

(f)

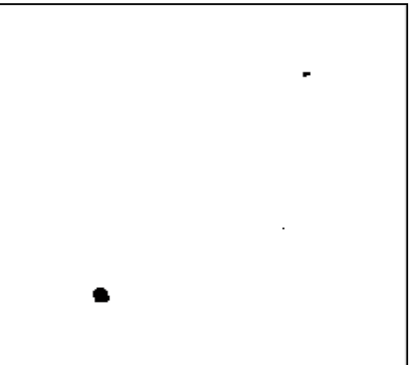

(c)

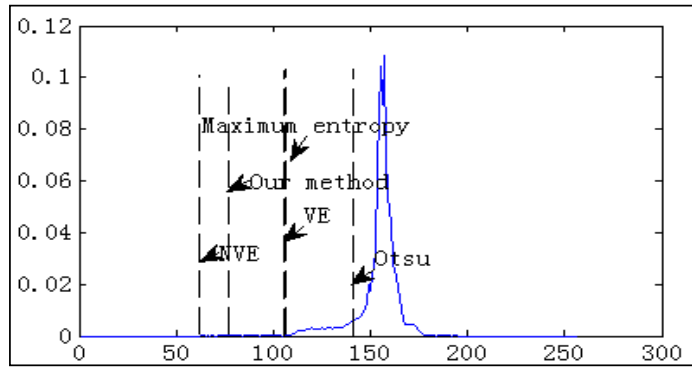

(g)

Fig.5 Segmentation results of a metal image: (a) Origin image, (b)-(f) the segmentation results of the Otsu, VE, NVE, maximum entropy and our method with threshold $T H=142,105,62,76,107$, respectively; (g) Histogram and threshold values.

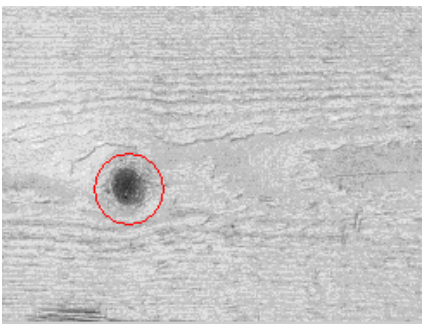

(a)

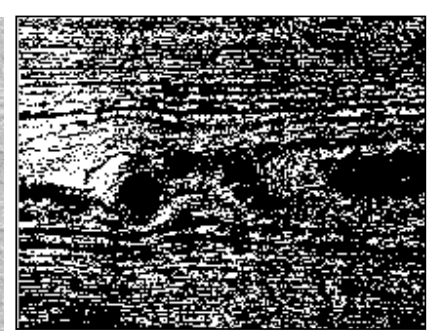

(b)

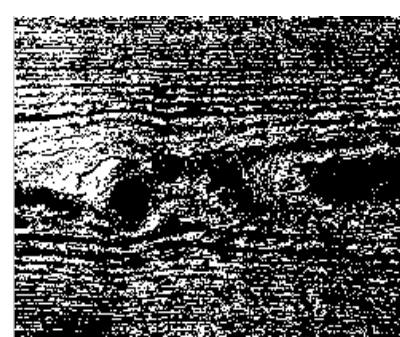

(c)

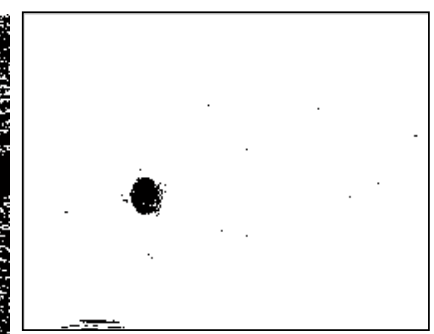

(d) 


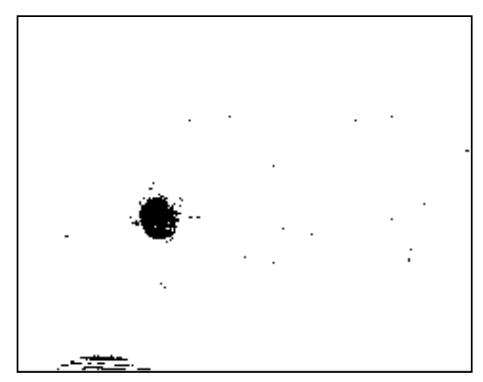

(e)

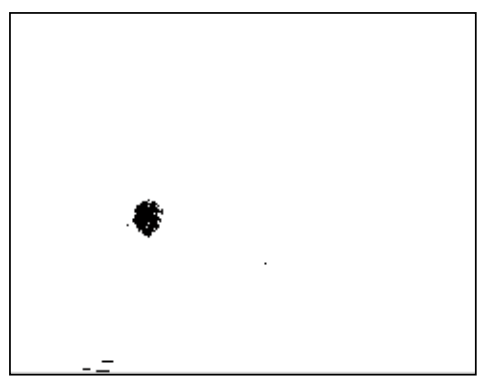

(f)

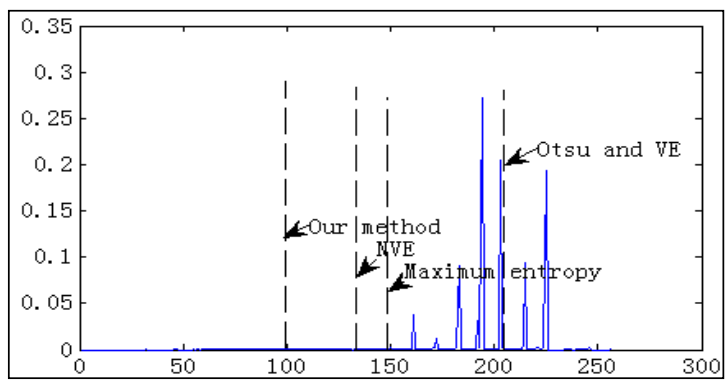

(g)

Fig.6 Segmentation results of a wood image: (a) Origin image; (b)-(f) the segmentation results of the Otsu, VE, NVE, maximum entropy and our method with threshold $T H=205,205,135,148,100$, respectively; (g) Histogram and threshold values.

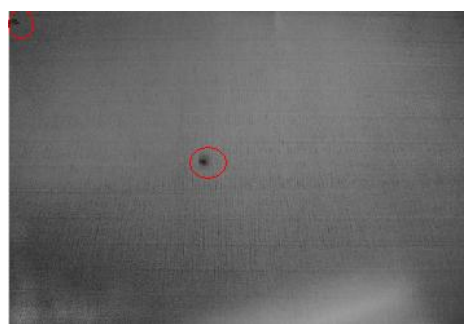

(a)

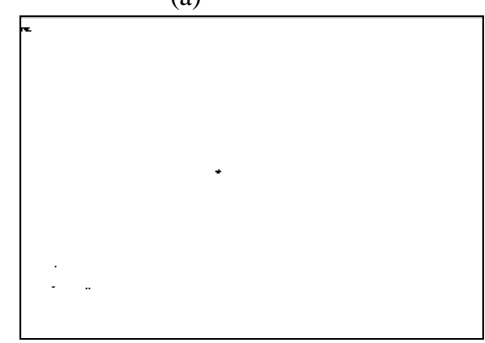

(e)

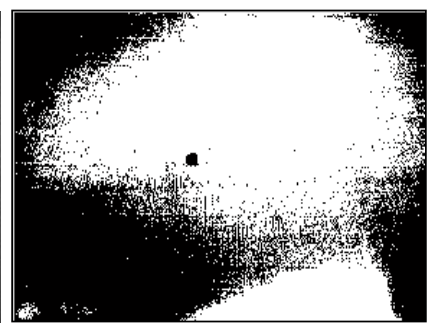

(b)

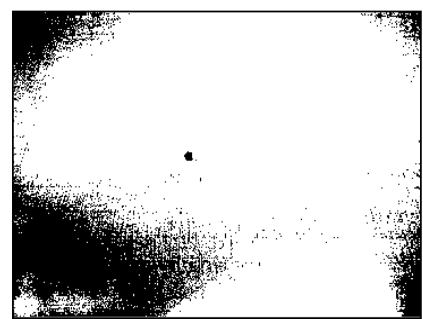

(c)

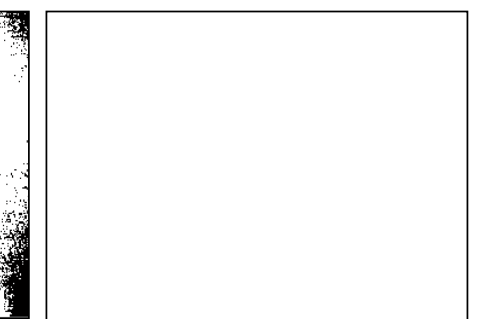

(d)

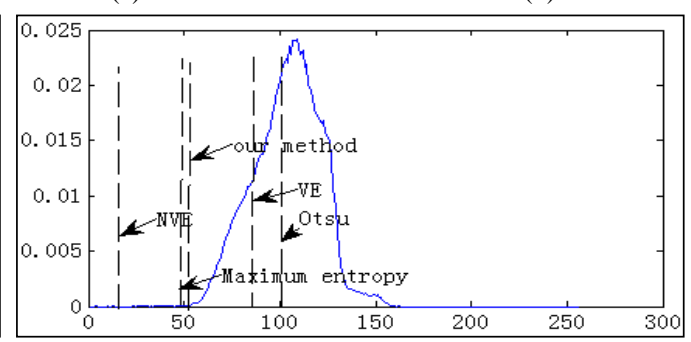

(g)

Fig.7 Segmentation results of a fabric image:(a) Origin image; (b)-(f) the segmentation results of the Otsu, VE, NVE, maximum entropy and our method with threshold $T H=100,86,17,49,51$, respectively; (g) Histogram and threshold values.

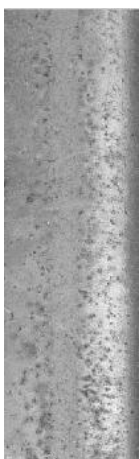

(a)

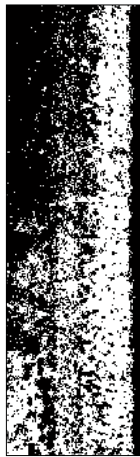

(b)

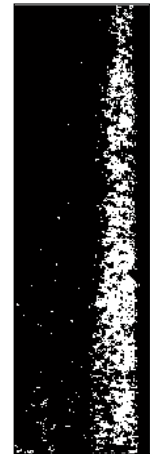

(c)

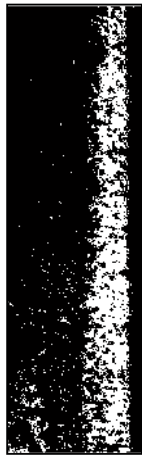

(e)

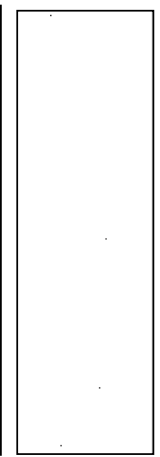

(f)

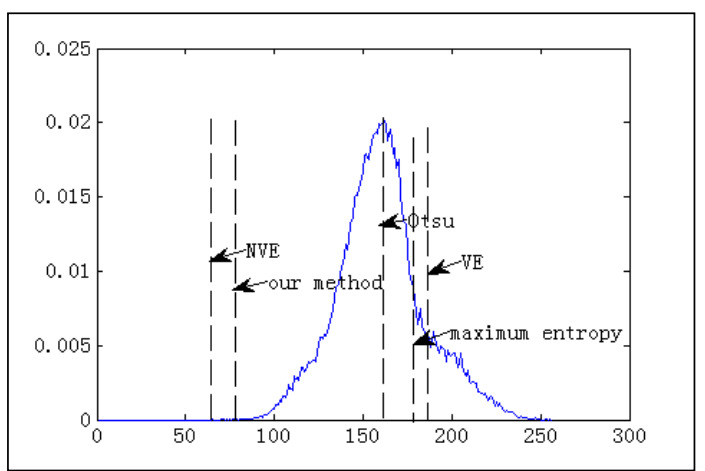

(g)

Fig.8 Segmentation results of defect-free rail image I: (a) Origin image; (b)-(f) the segmentation results of the Otsu, VE, NVE, maximum entropy and our method with threshold $T H=162,186,65,175,78$, respectively; (g) Histogram and threshold values;

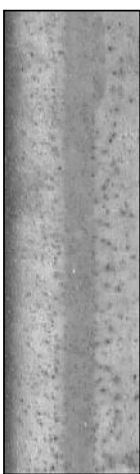

(a)

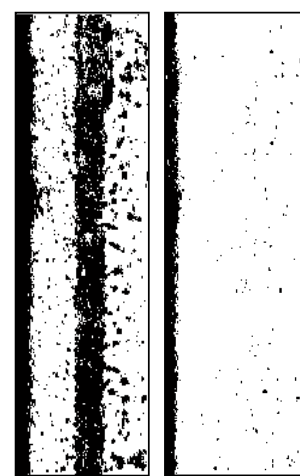

(b)

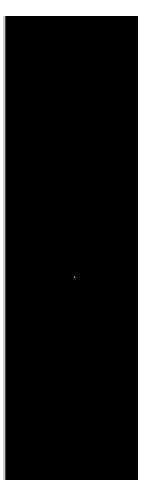

(d)

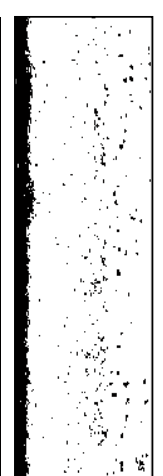

(e)

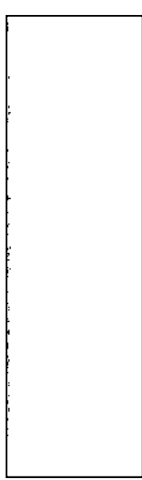

(f)

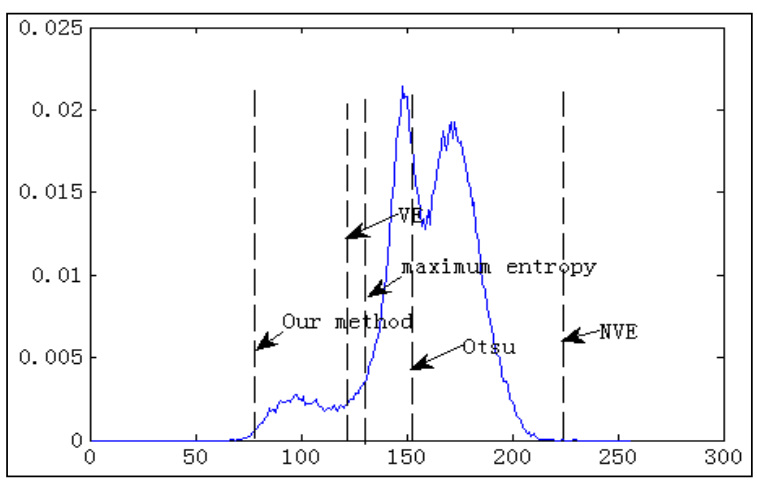

(g)

Fig.9 Segmentation result of defect-free rail image II: (a) Origin image, (b)-(f) the segmentation results of the Otsu, VE, NVE, maximum entropy and our method with threshold $T H=152,122,225,131,78$, respectively; (g) Histogram and threshold values 


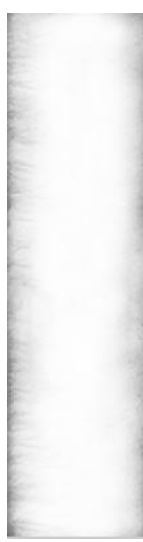

(b)

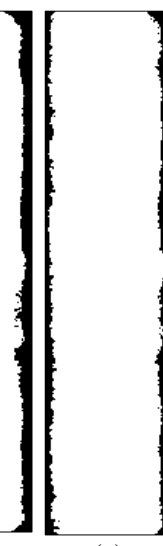

(c)

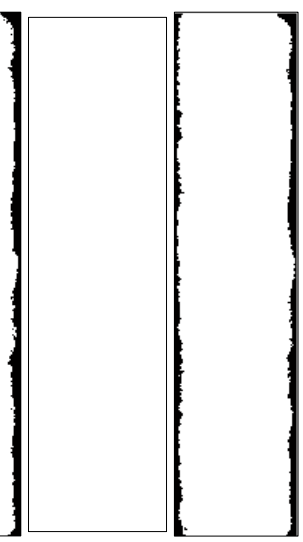

(d)

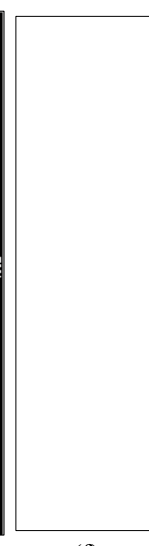

(f)

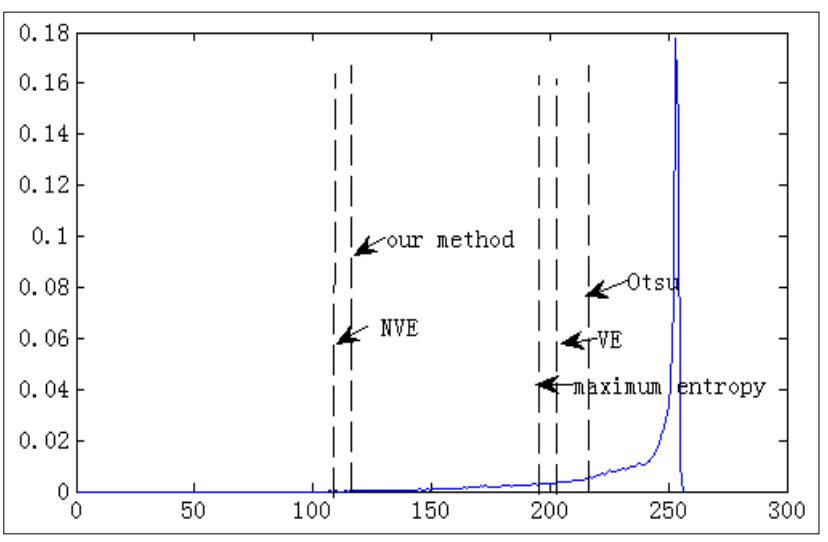

(g)

Fig.10 Segmentation result of defect-free rail image III: (a) Origin image, (b)-(f) the segmentation results of the Otsu, VE, NVE, maximum entropy and our method with threshold $T H=217,203,108,196,117$, respectively; (g) Histogram and threshold values;

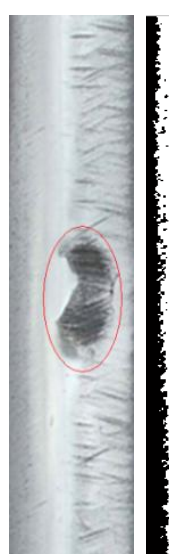

(a)

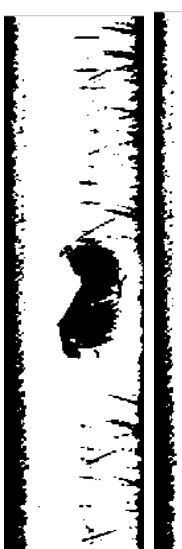

(b)

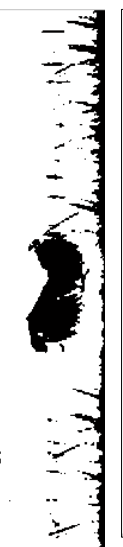

(c)

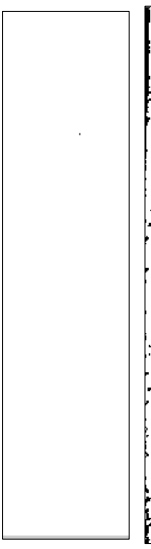

(d)

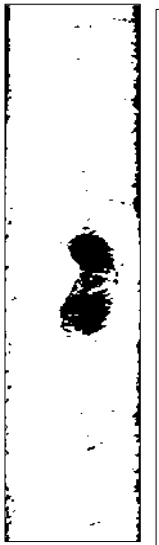

(e)

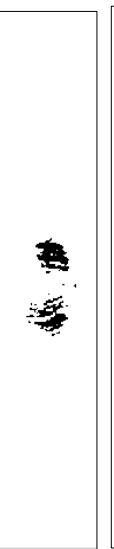

(f)

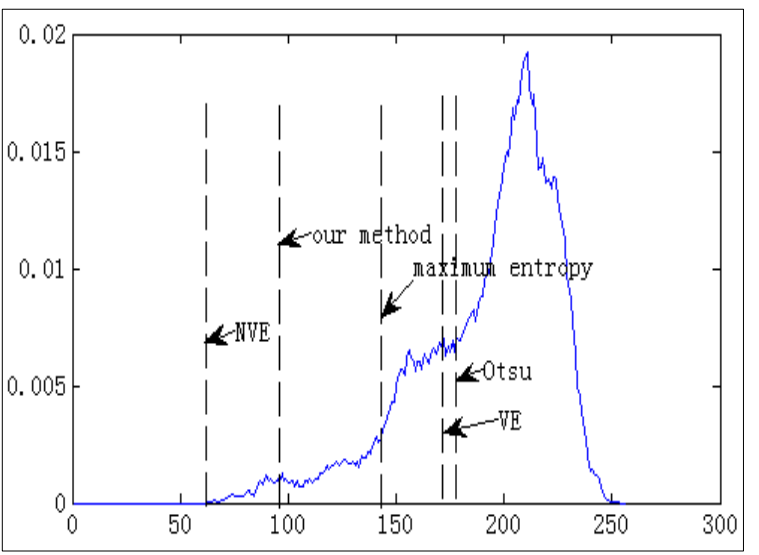

(g)

Fig.11 Segmentation result of defective rail image IV: (a) origin image, (b)-(f) the segmentation results of the Otsu, VE, NVE, maximum entropy and our method with threshold $T H=178,173,62,143,97$, respectively, $(\mathrm{g})$ histogram and threshold values

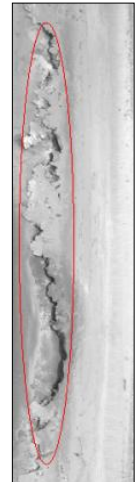

(a)

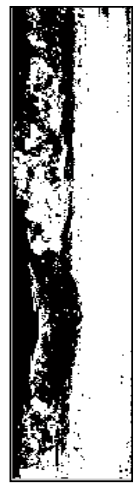

(b)

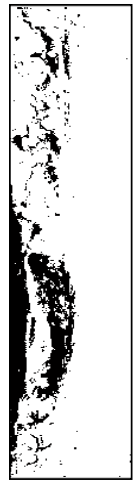

(c)

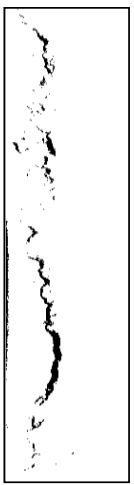

(d)

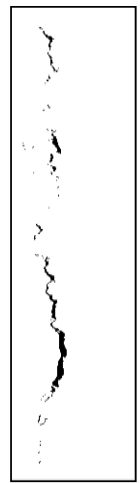

(e)

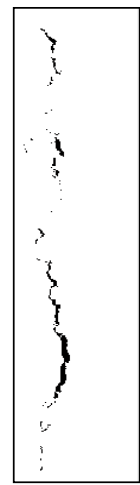

(f)

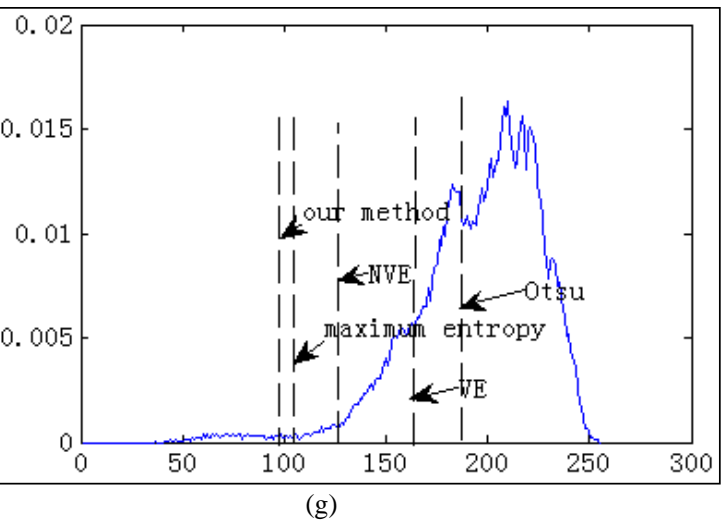

Fig.12 Segmentation results of rail image V: (a) Origin image, (b)-(f) the segmentation results of the Otsu, VE, NVE, maximum entropy and our method with threshold $T H=187,164,128,104,98$, respectively; (g) Histogram and threshold values

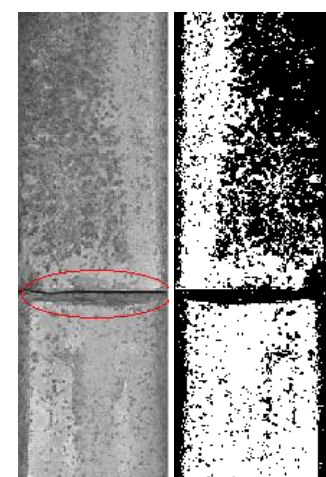

(a)

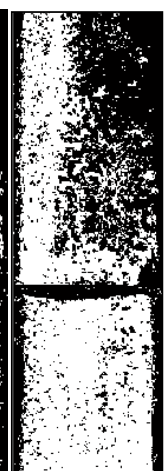

(c)

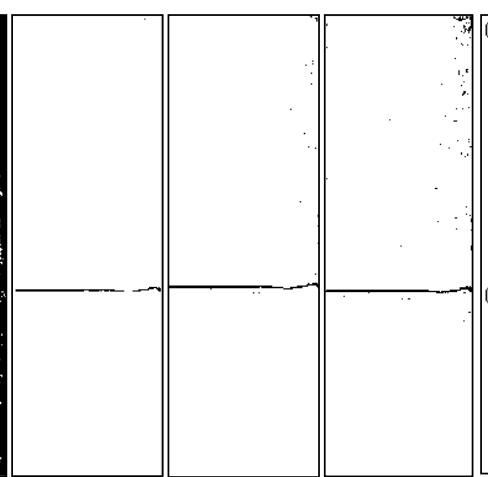

(d)

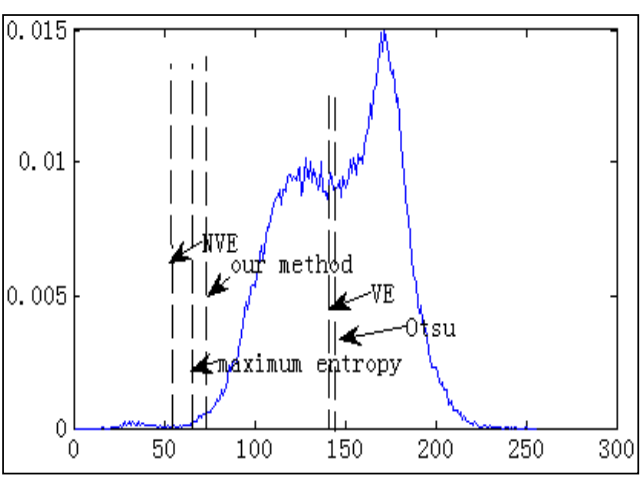

(g)

Fig.13 Segmentation results of rail image VI: (a) Origin image, (b)-(f) the segmentation results of the Otsu, VE, NVE, maximum entropy and our method with threshold $T H=142,140,55,65,73$, respectively; (g) Histogram and threshold values 


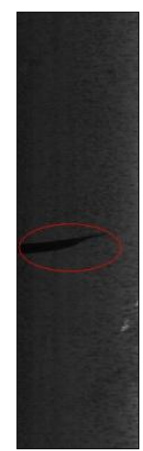

(a)

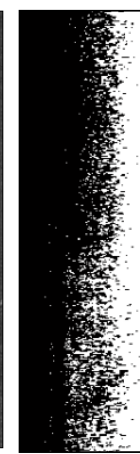

(b)

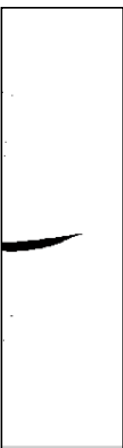

(c)

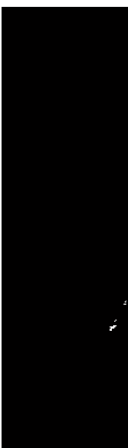

(d)

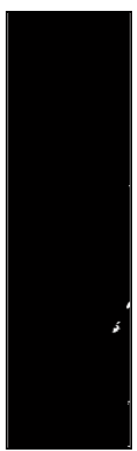

(e)

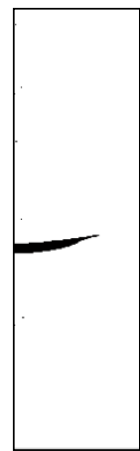

(f)

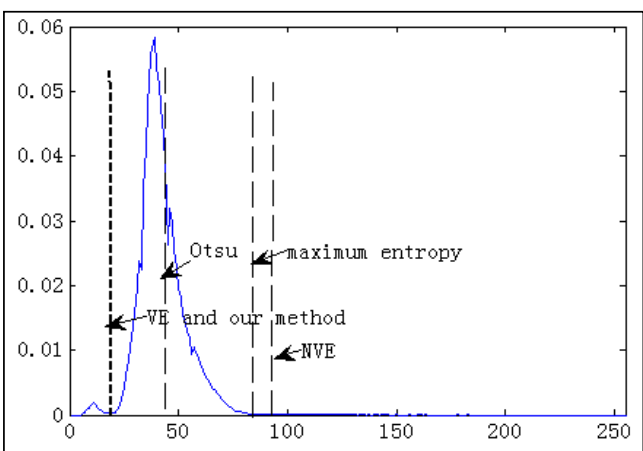

(g)

Fig.14 Segmentation results of rail image VII: (a) Origin image; (b)-(f) the segmentation results of the Otsu, VE, NVE, maximum entropy and our method with threshold $T H=44,19,93,86,20$, respectively; (g) Histogram and threshold values.

Table1. Misclassification errors of five methods for ten images

\begin{tabular}{lccccc}
\multicolumn{7}{c}{ Table1. Misclassification errors of five methods for ten images } \\
\cline { 2 - 6 } Application & \multicolumn{5}{c}{ Values of ME } \\
\cline { 2 - 6 } & Otsu & VE & NVE & $\begin{array}{c}\text { Maximum } \\
\text { entropy }\end{array}$ & Our method \\
\hline Metal image & 0.1091 & 0 & 0.0015 & 0.0012 \\
Wood image & 0.6894 & 0.6894 & 0.0051 & 0.0065 & 0 \\
Fabric image & 0.4166 & 0.1866 & 0.0045 & 0.0003 & 0.0005 \\
Rail image I & 0.5844 & 0.8663 & 0 & 0.8163 & 0.0225 \\
Rail image II & 0.4036 & 0.0923 & 1 & 0.1209 & 0 \\
Rail image III & 0.1775 & 0.1176 & 0 & 0.0955 & 0.0111 \\
Rail image IV & 0.2668 & 0.2230 & 0.0289 & 0.0602 & 0.0034 \\
Rail image V & 0.3525 & 0.1404 & 0.0133 & 0 & 0.0033 \\
Rail image VI & 0.4455 & 0.4272 & 0.0006 & 0 & 0 \\
Rail image VII & 0.6845 & 0 & 0.9881 & 0.9878 &
\end{tabular}

\subsection{Detection rate and false alarm rate of defects for the rail defect detection}

In Section 4.1, the ME value is used to evaluate the segmentation performance of images. However, only the ME value as the evaluation is not enough for the defect detection in visual inspection systems. Users usually concern DR and FAR more than ME values in practical applications. In addition, some images with small ME values may be not easy to generate correct segmentation results. For example, the segmentation results of the metal and fabric images in Fig. 5, 7, the NVE method obtains $\mathrm{ME}$ values close to 0 , however, it segments small defects into the background. In the vision inspection system, some noise may be detected as defects, but they can be ignored if the defects are very small.

A data set with 1000 rail images captured in the actual railways using a line-array $\mathrm{CCD}$ camera is constructed to comprehensively test the defects detection results. The data set consists of 177 images with at least one defect and 823 defectfree images. More defect-free than defective images are included because most rail surfaces are defect-free in the actual railway in service. Among these images, some rail surfaces are covered by rust, some smooth in center but dark in periphery regions. DR and FAR are widely used in pattern recognition and import performance indexes of vision inspection system for defect detection[33]. DR and FAR are described as follows:

$$
D R=\frac{\text { Number of defective samples correctly detected }}{\text { Total number of defective samples }}
$$

\section{$F A R=\frac{\text { Number of defect-free samples detected as defective }}{}$ Total number of defect-free samples}

After thresholding, a gray image becomes a binary image which contains only 0 and 255 two levels of black and white pixels. Computing the pixel number of every connective objects or detects, if the number is smaller than a given threshold, these objects are considered as noises, otherwise, they are defects.

Values of DR and FAR using the five methods for tested images are presented in Table 2. Otsu threshold biases toward the component with the larger between-class variance making the background and object variances a similar size. Therefore, the Otsu method detects all defect-free images as defective images. The NVE method gives a high DR, but also provides the high FAR as some defect-free images of rail surfaces covered by rust are detected as defects. The maximum entropy method does not obtain a high DR because some defects are hidden in rust. Our method provides a threshold value that locates at the left bottom rim of the histogram for defect-free images and the valley of two peaks for defective images. Therefore, our method obtains the highest DR and lowest FAR, which meets the requirement of a vision inspection system.

Table2. Comparison of DR and FAR for five methods

\begin{tabular}{cccccc}
\hline & VE & NVE & Otsu & $\begin{array}{c}\text { Maximum } \\
\text { entropy }\end{array}$ & $\begin{array}{l}\text { Our } \\
\text { method }\end{array}$ \\
\hline DR & $75.2 \%$ & $92.2 \%$ & $48.1 \%$ & $84.6 \%$ & $94.0 \%$ \\
FAR & $68.2 \%$ & $56.5 \%$ & $100 \%$ & $89.9 \%$ & $8.4 \%$ \\
\hline
\end{tabular}

4.3 Applications for classic images segmentation.

Besides defect detections using the WOV method, some 
classic images in digital image processing are also used to test the proposed WOV method. Three kinds of images with histograms that are close to unimodal, unimodal and bimodal distributions are tested. Figs. 15 to 17 show segmentation results and histograms for Moon, Rice and Circular images, respectively. The WOV method provides nearly perfect results for images with histograms that are close to unimodal or bimodal distributions, such as the moon and circular images, while it presents wrongly results for images with the histogram unimodal distribution as the threshold value locates at the left bottom rim of a histogram, such as the Rice image. The NVE method obtains better segmentation results than others for these three images.

The proposed WOV method obtains correct segmentation results for various defective images with small defects and defective-free images where their histograms are bimodal or close to the unimodal distribution, but it is incorrect for images with large defects or objects where their histograms are the unimodal distribution.

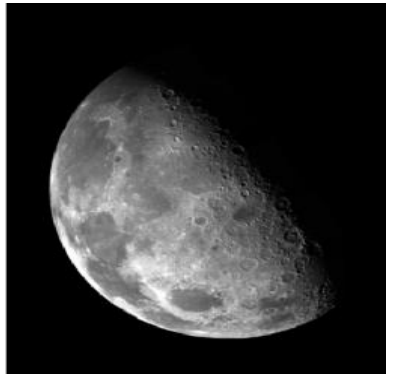

(a)

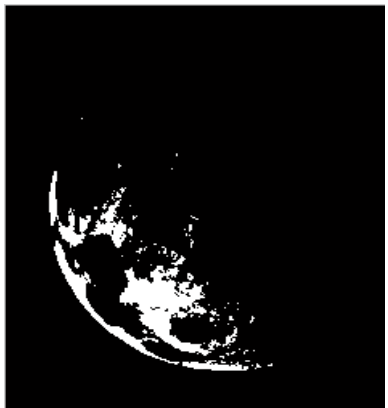

(e)

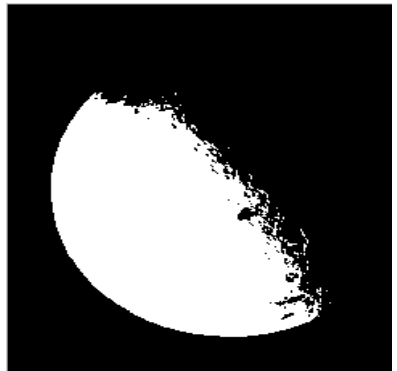

(b)

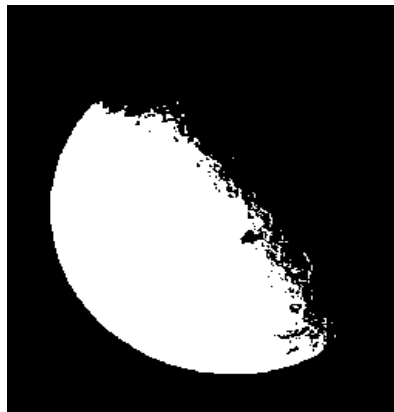

(f)

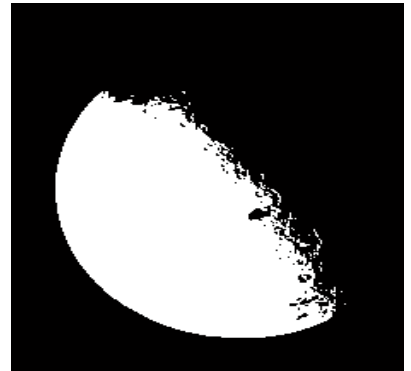

(c)

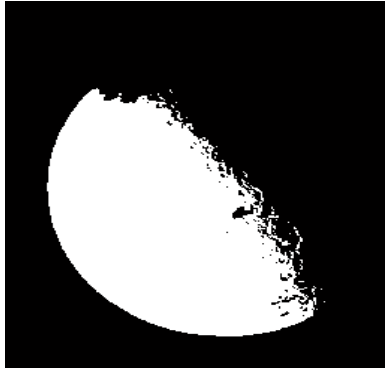

(d)

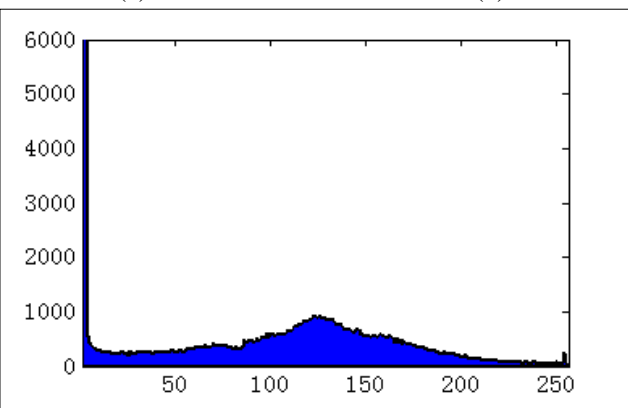

(g)

Fig.15 Segmentation results of Moon image with histogram close to unimodal distribution. (a) Origin image; (b)-(f) the segmentation results of Otsu, VE, NVE, maximum entropy and our method with threshold $T H=71,70,66,172,69$, respectively; (g) histogram of Moon image.

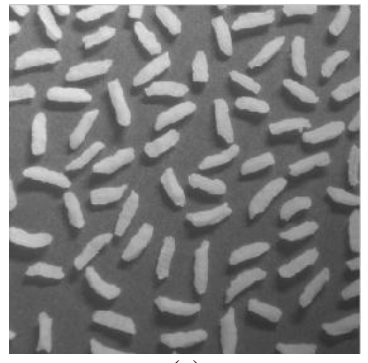

(a)

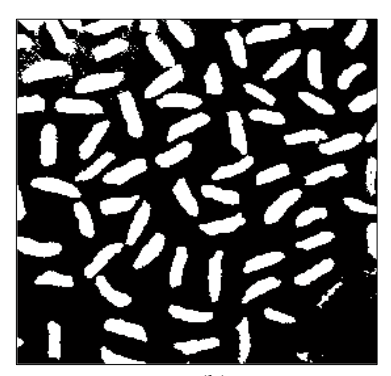

(b)

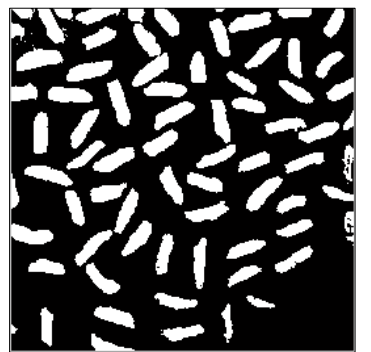

(c)

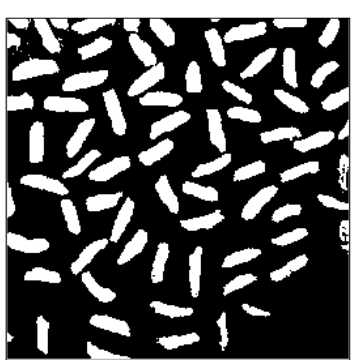

(f)

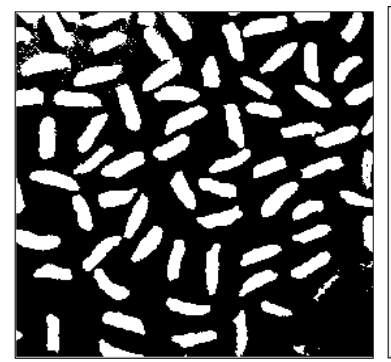

(e)

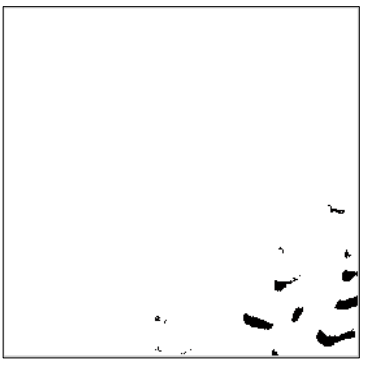

(f)

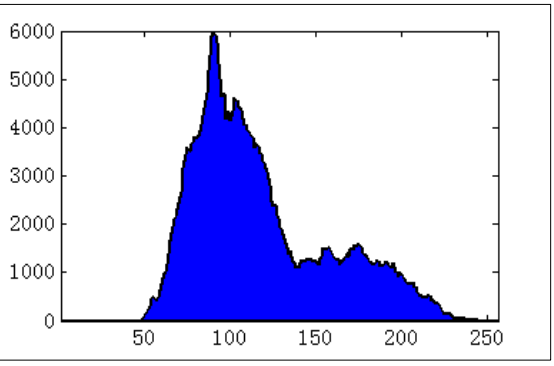

(g)

Fig.16 Segmentation results of Rice image with histogram unimodal distribution. (a) Origin image,(b)-(f) the segmentation results of Otsu, VE, NVE, maximum entropy and our method with threshold $T H=134,138,141,134,58$, respectively, $(\mathrm{g})$ histogram of Rice image. 


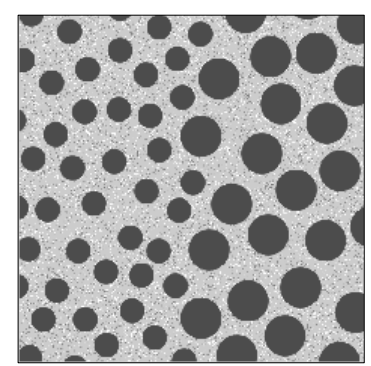

(a)

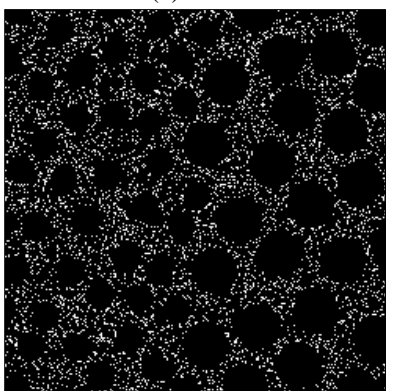

(e)

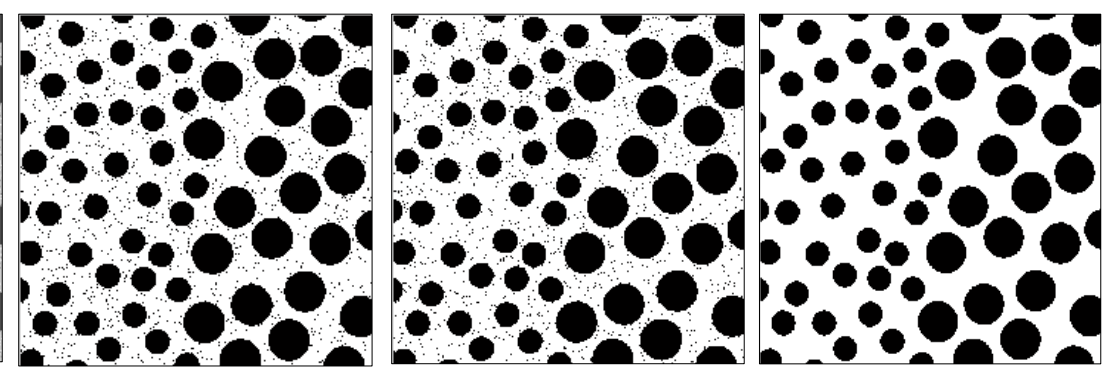

(b)

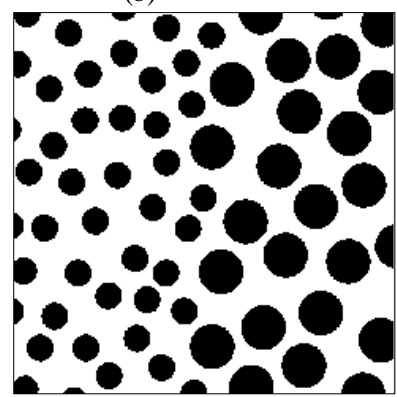

(f) (c)

(d)

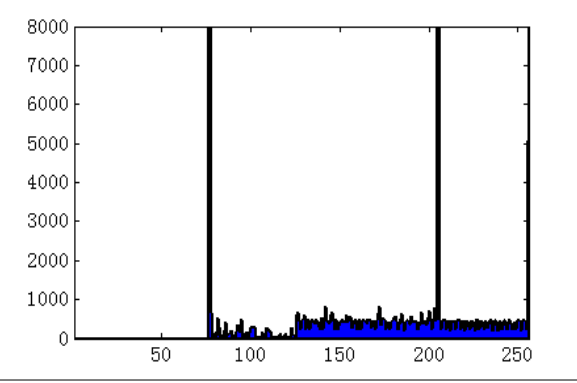

(g)

Fig.17 Segmentation results of Circular image with histogram bimodal distribution. (a) Origin image; (b)-(f) the segmentation results of Otsu, VE, NVE, maximum entropy and our method with threshold $T H=142,141,117,92,118$, respectively; (g) histogram of Circular image.

\subsection{The influence of weighted $\omega$ in defect detection}

As described in above, different values of weight can lead to different threshold values. A smaller $\omega$ will result in a smaller threshold and a larger $\omega$ will make the threshold close to the Otsu threshold. A group of weights $\omega=0,0.2,0.4,0.6,0.8$, is constructed to detect defects for the 1000 rail images and compared to $\omega=P_{0}(t)$. DR and FAR are used to measure the detection results. When $\omega=0$, Eq. (9) can be written as $Y_{b}(t)=P_{1}(t)\left(u_{1}(t)\right)^{2}$, the threshold value is only decided by the background variance. The results of DR and FAR are listed in Table 3. It can be concluded that when $\omega=0$, the FAR is the lowest as the threshold is the smallest, and when $\omega=P_{0}(t)$, the DR is the highest. However, the DR is more important than the FAR, therefore, $\omega=P_{0}(t)$ is a better selection for the defect detection applications. In addition, the invariable value of weight, such as $\omega=0$, cannot satisfy requirements for various images when images vary from different products. An adaptive weight is therefore a better selection for the machine vision inspection.

Table3.Comparison of DR and FAR for $\omega=0,0.2,0.4,0.6,0.8, P_{0}(t)$

\begin{tabular}{ccccccc}
\multicolumn{6}{c}{ Table3.Comparison of DR and FAR for $\omega=0,0.2,0.4,0.6,0.8, P_{0}(t)$} \\
\hline & $\omega=0$ & $\omega=0.2$ & $\omega=0.4$ & $\omega=0.6$ & $\omega=0.8$ & $\omega=P_{0}(t)$ \\
\hline DR & $90.2 \%$ & $93.2 \%$ & $85.6 \%$ & $73 \%$ & $70.0 \%$ & $94 \%$ \\
FAR & $2.2 \%$ & $10.5 \%$ & $23.5 \%$ & $59.9 \%$ & $77.2 \%$ & $8.4 \%$ \\
\hline
\end{tabular}

\section{Conclusions}

In this paper, an improved Otsu method, namely the WOV method, is presented to automatically select the optimal threshold value for the defect detection. A parameter ranged from 0 to 1 is used as a weight on the object variance of between-class. The weight that equals the cumulative probability of the defect occurrence is a better selection for defect detection applications. The automatic weighting method ensures that the threshold will always be a value that locates at two peaks or at the left bottom rim of a single peak. Comparing the proposed method with the maximum entropy, Otsu, VE and NVE methods, the proposed WOV method obtains better segmentation results for the defect detection. The VE threshold value locates at a local valley of the histogram, and gives the wrong threshold for most of defect-free images or detects the background as defects. The NVE threshold value locates at the bottom rim of a single peak, so it wrongly segments the background as defects for some of defect-free images. The maximum entropy method successfully segments most of defective images, but fails for defect-free images, which leads to a high FAR for the defect detection. It is demonstrated in experiments that the proposed WOV method can be used for various defect detection applications, such as the contamination inspection, wood node detection, and rail surface crack detection. The proposed WOV method gets $94 \%$ DR and $8.4 \%$ FAR of the defect detection for tested rail images. In addition, the WOV method is simple and easy to implement, it can be used to the real time defect inspection. As the proposed WOV threshold value locates at the left bottom rim of a single peak, it is essential for a low FAR of the defect detection. However, it is incorrect for images with large defects or objects where their histograms are the unimodal distribution, such as the Rice image in Fig.16. In the future, more work will be devoted to the single peak image segmentation.

\section{Acknowledgements}

This work was supported by National Science Foundation of China (grant no. 51065021).

\section{References}

[1] J. Atzlesberger, B.G. Zagar, R. Cihal, M. Brummayer, P. Reisinger, Subsurface defect detection in a steel sheet, Measurement Science \& Technology, 24 (2013).

[2] F. Xie, L. Xiao, Y. Lu, H. Zhang, Research on an eddy current sensor used for steel ball surface defect detection, Transducer and Microsystem Technology, 31 (2012) 34-35,39.

[3] W.-b. Li, C.-h. Lu, J.-c. Zhang, A local annular contrast based real-time inspection algorithm for steel bar surface defects, Applied Surface Science, 258 (2012) 6080-6086.

[4] K.K. Patel, A. Kar, S. Jha, M. Khan, Machine vision system: a tool for quality inspection of food and agricultural products, Journal of food science and technology, 49 (2012) 123-141.

[5] K. Song, Y. Yan, A noise robust method based on completed local binary patterns for hot-rolled steel strip surface defects, Applied Surface Science, 285 (2013) 858-864.

[6] S.S. Al-amri, N.V. Kalyankar, Image segmentation by using threshold techniques, arXiv preprint arXiv:1005.4020, (2010). 
[7] H. Yazid, H. Arof, H. Yazid, Automated thresholding in radiographic image for welded joints, Nondestructive Testing and Evaluation, 27 (2012) 69-80.

[8] P. Sathya, R. Kayalvizhi, Optimal multilevel thresholding using bacterial foraging algorithm, Expert Systems with Applications, 38 (2011) $15549-15564$.

[9] L. Qingyong, Y. Huang, Z. Liang, L. Siwei, Thresholding Based on Maximum Weighted Object Correlation for Rail Defect Detection, IEICE TRANSACTIONS on Information and Systems, 95 (2012) 1819-1822.

[10] J. Funck, Y. Zhong, D. Butler, C. Brunner, J. Forrer, Image segmentation algorithms applied to wood defect detection, Computers and electronics in agriculture, 41 (2003) 157-179.

[11] M. Shi, R. Fu, Y. Guo, S. Bai, B. Xu, Fabric defect detection using local contrast deviations, Multimedia Tools and Applications, 52 (2011) 147-157.

[12] D.-M. Tsai, J.-Y. Luo, Mean shift-based defect detection in multicrystalline solar wafer surfaces, Industrial Informatics, IEEE Transactions on, 7 (2011) 125-135.

[13] S. Ghorai, A. Mukherjee, M. Gangadaran, P.K. Dutta, Automatic defect detection on hot-rolled flat steel products, Instrumentation and Measurement, IEEE Transactions on, 62 (2013) 612-621.

[14] M. Sezgin, Survey over image thresholding techniques and quantitative performance evaluation, Journal of Electronic imaging, 13 (2004) 146-168.

[15] N. Otsu, A threshold selection method from gray-level histograms, Automatica, 11 (1975) 23-27.

[16] S. Nashat, A. Abdullah, M. Abdullah, Unimodal thresholding for Laplacian-based Canny-Deriche filter, Pattern Recognition Letters, 33 (2012) 1269-1286.

[17] Q. Hu, Z. Hou, W.L. Nowinski, Supervised range-constrained thresholding, Image Processing, IEEE Transactions on, 15 (2006) 228-240.

[18] Y. Qiao, Q. Hu, G. Qian, S. Luo, W.L. Nowinski, Thresholding based on variance and intensity contrast, Pattern Recognition, 40 (2007) 596-608.

[19] H.-F. Ng, Automatic thresholding for defect detection, Pattern Recognition Letters, 27 (2006) 1644-1649.

[20] J.-L. Fan, B. Lei, A modified valley-emphasis method for automatic thresholding, Pattern Recognition Letters, 33 (2012) 703-708.
[21] X. Xu, S. Xu, L. Jin, E. Song, Characteristic analysis of Otsu threshold and its applications, Pattern Recognition Letters, 32 (2011) 956961.

[22] W. Xue-guang, C. Shu-hong, An improved image segmentation algorithm based on two-dimensional Otsu method, Inf. Sci. Lett, 1 (2012) $77-83$

[23] L. Jianzhuang, L. Wenqing, T. Yupeng, Automatic thresholding of graylevel pictures using two-dimension Otsu method, in: Circuits and Systems, 1991. Conference Proceedings, China., 1991 International Conference on, IEEE, 1991, pp. 325-327.

[24] Q. Chen, L. Zhao, J. Lu, G. Kuang, N. Wang, Y. Jiang, Modified twodimensional Otsu image segmentation algorithm and fast realisation, IET image processing, 6 (2012) 426-433.

[25] L. Yi-bing, M. Ting, L. Ao, Two-Dimensional Otsu Image Segmentation Algorithm Based on the Particle Swarm Optimization Algorithm, in: Future Control and Automation, Springer, 2012, pp. 115-120.

[26] W.A. Yasnoff, J.K. Mui, J.W. Bacus, Error measures for scene segmentation, Pattern Recognition, 9 (1977) 217-231.

[27] H.Y. Ngan, G.K. Pang, N.H. Yung, Automated fabric defect detection-a review, Image and Vision Computing, 29 (2011) 442-458.

[28] J. Kapur, P.K. Sahoo, A. Wong, A new method for gray-level picture thresholding using the entropy of the histogram, Computer Vision, Graphics, and Image Processing, 29 (1985) 273-285.

[29] N. Nacereddine, L. Hamami, N. Oucief, Non-parametric histogrambased thresholding methods for weld defect detection in radiography, World Academy of Science, Engineering and Technology, 9 (2005) 213-217.

[30] Q. Lin, C. Ou, Tsallis entropy and the long-range correlation in image thresholding, Signal Processing, 92 (2012) 2931-2939.

[31] Y. Zou, H. Liu, E. Song, Z. Huang, Image bilevel thresholding based on multiscale gradient multiplication, Computers \& Electrical Engineering, 38 (2012) 853-861.

[32] M. Beauchemin, Image thresholding based on semivariance, Pattern Recognition Letters, 34 (2013) 456-462.

[33] J. Li, X. Rao, F. Wang, W. Wu, Y. Ying, Automatic detection of common surface defects on oranges using combined lighting transform and image ratio methods, Postharvest Biology and Technology, 82 (2013) 59-69. 